\title{
Editorial
}

Chris H. Kehler MD FRCPC

\section{Evolution of patient care in the early postoperative period}

The transfer of a patient from the operating room to the recovery room is an event that we regard as standard practice in anaesthesia. This was not always the case. Although the need for specialized postoperative patient care was recognized in the early 1800's, the early 1900's found only a few large American hospitals with designated "recovery rooms." In the majority of hospitals, patients were sent directly to their wards from the operating room. World War II brought an increase in the number of critically ill surgical patients and the formation of postanaesthesia recovery areas occurred in many hospitals. The first Canadian recovery room was created by Dr. Harold Griffith in 1943. Of the many contributions that he made to patient care, this was one of his greatest.

Since the days of the early recovery rooms, advances in surgical techniques have increased the demand for specialized perioperative care. A 1981 review of the design of a modem recovery room ${ }^{\natural}$ included the following definition:

"The recovery room is an intensive care unit designed for the care of patients from the time they leave the operating room until they are sufficiently recovered from the physiological derangements induced by anaesthesia and surgery to be transferred to other areas appropriate to their needs."

In the traditional sense, the above-mentioned physiological derangements are regarded as unconsciousness and cardiorespiratory depression in the patient who has had a general anaesthetic, and the absence of sensation and sympathetic tone in the

Department of Anesthesia, Health Sciences Centre, University of Manitoba, Winnipeg, Manitoba R3E $0 Z 3$. patient who has had a regional anaesthetic. Once these functions have returned to their preoperative state, and no major surgical complications have ensued, the patient is retumed to the surgical ward. As mentioned, this tradition of postoperative patient care has been in effect since the early 1940's.

With improvement in our understanding of the physiological changes that occur in the perioperative period we are better able to identify patients who are at an increased risk of developing potentially life threatening postoperative problems. By anticipating these problems, optimizing preoperative status and closely monitoring perioperative cardiorespiratory function we can successfully manage such high-risk patients. In the process of trying to provide the best possible medical care, we continually increase the demands on postoperative patient care areas. What facilities are necessary to meet these demands? The options available in most hospitals are the recovery room or the intensive care unit. Unfortunately, the capacity of many intensive care units is already being pushed to the limit. This problem presents us with the challenge of objectively identifying those patients who require postoperative admission to the intensive care area. It also presents us with a group of patients who may not fulfil intensive care admission criteria but require a higher level of care than they could receive on the surgical ward. It is the care of these patients that is addressed by Landow in this issue of the Journal. ${ }^{2}$

Landow's approach, to create an intermediate care facility in the recovery room, is one answer to the needs of high-risk patients who do not meet the criteria for intensive care admission. While I can see the rationale in such an approach, there are a few points that must be addressed. Firstly, it appears that Landow is the only anaesthetist involved in the management of patients in his intermediate care 
facility. While it is necessary to have a director to ensure that standards of care are being met, it seems unrealistic to have only one person to provide continuous supervision and support for a four-bed critical care unit.

In his description of nursing coverage, Landow correctly points out that recovery room nurses are valued members of the critical care staff. Although nurse-to-patient ratios are addressed, no mention is made of the actual number of nurses in the intermediate care unit. Clearly, this will be one of the limiting factors in the creation of such a unit. Provision of staff would have to account for a nurse to patient ratio of $1: 1$ to $1: 2$ in the intermediate care area, as well as providing nurses to care for patients having emergency surgery. Depending on how active the emergency $O R$ is, the staffing requirement may be quite substantial.

Admission criteria to an intermediate care area will depend upon the patient's underlying cardiovascular and pulmonary status, the proposed surgical procedure and the available intensive care facilities. The creation of such criteria should include input from anaesthetists, surgeons, intensivists and the medical director and head nurse of the recovery room. Clearly, the division between intensive care candidates and intermediate care candidates will vary between hospitals. The most important point is that objective guidelines are set and communication occurs between those responsible for a given patient's care.

While I have little argument with Landow's general protocols, I can not agree with the use of intramuscular narcotic as the method of analgesia routinely provided in the recovery room. To deny a patient a more rapid, titratable route of narcotic administration in an area where patients are being so closely monitored does not seem reasonable. Landow seems to have done a great deal to improve patient care in his hospital; a change in policy regarding administration of intravenous narcotic by recovery room nurses would add to his improvements.

Since its creation, the recovery room has evolved into a highly sophisticated short-term intensive care area. Patient turnover is relatively high, potentially life-threatening problems are always present and the need for vigilance applies to the recovery room just as it does in the operating room. With an ever-increasing demand to provide care for high- risk surgical patients, our intensive care units and recovery rooms are constantly being challenged. In meeting this challenge, these facilities are being forced to expand and improve. A logical step in this evolution is to designate an area where a patient may receive optimal management without the need to wait in line for an intensive care bed. In his description of a modern recovery room, Landow outlines some of the considerations in establishing an intermediate care facility. Although the approach to establishing such a facility will vary between centres, the basic principles remain the same. As anaesthetists, we have the responsibility to ensure the best possible perioperative care for our patients. In 1943, Dr. Griffith followed this philosophy in establishing the first Canadian recovery room. More than 40 years later the process of evolution continues; a reassuring sign that we are always in search of a better way to provide patient care.

\section{L'évolution des soins medicaux dans la periode post- opératoire précoce}

Le transfert du patient de la salle d'operation à la salle de réveil est une événement actuellement considéré commun en pratique anesthésique. Ceci n'a pas toujours été le cas. Même si l'importance de soins médicaux post-opératoires spécialisés a été reconnue dès le début des années 1800 , ce n'est que vers le début des années 1900 qu'on commença à désigner dans quelques grands hôpitaux américains des endroits reconnus comme étant des "salles de réveil". Dans la majorité des hôpitaux, les patients étaient renvoyés directement à leur étage à partir de la salle d'opération. La deuxième guerre mondiale augmenta le nombre de patients chirurgicaux critiquement malades et déclencha la création de locaux de salle de réveil dans plusieurs hôpitaux. La première salle de réveil canadienne a été créé par le 
docteur Harold Griffith en 1943. Parmi ses multiples contributions aux soins du malade, cette dernière a été sa plus importante.

L'amélioration des techniques chirurgicales a, depuis cette période initiale, augmenté la demande pour des soins péri-opératoires spécialisés. En 1981 une revue du concept d'une salle de réveil moderne' comprenait la définition suivante:

"La salle de réveil est une unité de soins intensifs établie pour les patients dès leur sortie de la salle d'opération jusqu'au rétablissement des altérations physiologiques induites par l'anesthésie et la chirurgie permettant ainsi un transfert sécuritaire aux autres locaux appropriés à leurs besoins."

Au sens traditionnel, les altérations physiologiques susmentionnées sont considérées comme étant l'inconscience et la dépression cardiorespiratoire chez un patient ayant subi une anesthésie générale, et l'absence de sensibilité et de tonus sympathique chez le patient ayant subi une anesthésie régionale. Une fois ces fonctions rétablies à leur état préopératoire et qu'aucune complication chirurgicale n'a été déclenchée, le patient est retourné à l'unité chirurgicale. Comme mentionné cette tradition des soins médicaux post-opératoires a été établie depuis les débuts des années 1940 .

Avec l'amélioration de notre compréhension des changements physiologiques pouvant survenir en période péri-opératoire on est plus capable d'identifier les patients à risque élevé susceptibles de développer des problèmes post-opératoires potentiellement mortels. En anticipant ces problèmes, optimisant l'état pré-opératoire et surveillant la fonction cardionespiratoire péri-opératoire on peut altérer notre conduite afin de faire face avec succès à ces patients à haut risque. Dans le processus du choix des soins médicaux optimaux on a augmenté continuellement la demande dans le secteur des soins post-opératoires. Quelles sont les facilités nécessaires afin de remplir ces demandes? Les options disponibles dans la majorité des hôpitaux sont la salle de réveil et l'unité des soins intensifs. Malheureusement la capacité de plusieurs de ces unités de soins intensifs est déjà poussée à la limite. Ce problème nous oblige à identifier d'une façon objective les patients qui requièrent l'admission à l'unité des soins intensifs. Aussi ceci nous oblige à faire face à un groupe de patients qui ne remplissent pas les critères d'admission aux unités de soins intensifs mais qui requièrent un niveau de soins pour le moins supérieur à celui qu'ils reçoivent à l'unité chirurgicale. C'est le soin de ces patients qui est considéré par le docteur Landow dans cette émission du Journal. ${ }^{2}$

L'approche de Landow, de créer des locaux de soins intermédiaires dans la salle de réveil est une réponse aux besoins de ces patients à risque qui ne remplissent pas les critères d'admission aux soins intensifs. Alors que je vois la logique de cette approche plusicurs points doivent être considérés. Premièrement il apparaît que le docteur Landow est le seul anesthésiste impliqué dans le traitement de ces patients de cette unité de soins intermédiaires. Alors qu'il est nécessaire d'avoir un directeur afin d'assurer que les normes de soins sont appliquées il est impracticable pour une personne de foumir la surveillance continue ainsi que le support de l'unité de soins intensifs de quatre lits.

Dans sa description des soins infirmiers le docteur Landow soulève à juste titre le point que le personnel infirmier des salles de réveil sont des membres appréciés de l'équipe des soins intensifs. Même si le taux personnel infirmier sur patient est soulevé aucune mention n'est faite du nombre actuel du personnel infirmier à l'unité de soins intermédiaires. Certainement ceci sera un facteur limitant dans la création d'une telle unité. Lors de la formation de l'équipe on devra tenir compte d'un taux personnel infirmier sur patient de 1:1 à 1:2 dans les locaux des soins intermédiaires en même temps que prévoir du personnel infirmier pouvant prendre soin des patients devant subir une chirurgie d'urgence. Selon l'activité de la salle d'opération d'urgence un tel engagement du personnel infirmier peut être assez substantiel.

Les critères d'admission à l'unité des soins intermédiaires dépendront de l'état cardiovasculaire et pulmonaire sous-jacent du patient, la procédure chirurgicale envisagée et la disponibilité des facilités aux soins intensifs. La création de tels critères doit impliquer les anesthésistes, chirurgiens, les intensivistes, et le directeur médical ainsi que l'infirmière chef de la salle de réveil. Il est clair que la séparation entre les candidats susceptibles d'être admis aux soins intensifs et ceux susceptibles d'être admis aux soins intermédiaires peut varier d'un hôpital à l'autre. Le point le plus important 
sera d'établir les lignes directrices objectives et des voies de communication entre les responsables des soins du patient.

Alors que je conteste en général peu les protocoles du docteur Landow, je ne peux admettre l'utilisation de narcotiques par voie intramusculaire comme méthode d'analgésie de routine dans la salle de réveil. Refuser au patient une méthode rapide, titrable, d'administration de narcotiques alors que le patient est surveillé de façon étroite ne semble pas raisonnable. Il paraît que le docteur Landow a amélioré beaucoup les soins du patient dans son hôpital; un changement de politique concernant l'administration de narcotique par voie intraveineuse par l'équipe des soins infirmiers à la salle de réveil ajouterait à ces améliorations.

Depuis sa création, la salle de réveil s'est transformée en une région de soins intensifs à court terme hautement sophistiquée. Le roulement des patients est relativement élevé, des problèmes pouvant en présence mettre en danger la vie des patients sont toujours présents et la nécessité d'une vigilance s'applique à la salle de réveil autant qu'elle s'applique en salle d'opération. Avec une demande toujours croissante de foumir des soins pour les patients chirurgicaux à haut risque, nos soins intensifs et salles de réveil sont toujours constamment en demande. Afin de répondre à cette demande ces facilités sont obligées de s'expandre et de s'améliorer. Au cours de ce changement il apparaît qu'il y a une nécessité pour des locaux de soins intermédiaires où les patients à haut risque peuvent recevoir des soins post-opératoires optimaux sans occuper des locaux nécessaires pour des patients à risque beaucoup plus élevé. Une réponse à cette situation est celle décrite par Landow. Nous espérons que d'autres formuleront leurs opinions afin d'ajouter au peu de connaissance déjà publiée à ce sujet.

\section{References}

1 Warfield $C A$, Warfield $G R$. The postanesthetic recovery room. Int Anes Clin 1981; 19:63-75.

2 Landow $L$. The recovery room in the $1980^{\prime} \mathrm{s}$. Can Anaesth Soc J 1986; 33: 349-51. 